\title{
Intrauterine Undernourishment Alters TH1/TH2 Cytokine Balance and Attenuates Lung Allergic Inflammation in Wistar Rats
}

\author{
Maristella A. Landgraf ${ }^{1,2 a}$ Richardt G. Landgrafa ${ }^{2 a}$ Reinaldo C. Silva ${ }^{3}$ Patrícia \\ Semedo $^{3}$ Niels O. S. Câmara ${ }^{1,3 b}$ Zuleica B. Fortes ${ }^{4 b}$ \\ ${ }^{1}$ Laboratorio de Imunologia dos Transplantes, Departamento de Imunologia, Instituto de Ciências \\ Biomédicas, Universidade de São Paulo, São Paulo, nstituto de Ciências Ambientais, Químicas e \\ Farmacêuticas, Universidade Federal de São Paulo - Campus Diadema, São Paulo, ${ }^{3}$ Laboratorio de \\ Imunologia Clínica e Experimental, Divisão de Nefrologia, Universidade Federal de São Paulo, São \\ Paulo, ${ }^{4}$ Departamento de Farmacologia, Instituto de Ciências Biomédicas, Universidade de São Paulo, \\ São Paulo, ${ }^{a, b}$ These authors contributed equally to this work
}

\section{Key Words}

Asthma • Intrauterine undernutrition • Inflammation • Cytokines • GATA-3 • T-bet • Th1/Th2 balance

\begin{abstract}
IL-4 produced by Th2 cells can block cytokine production by Th1 cells, and Th1 IFN- $\gamma$ is known to counterregulate Th2 immune response, inhibiting allergic eosinophilia. As intrauterine undernutrition can attenuate lung inflammation, we investigated the influence of intrauterine undernourishment on the Th1/Th2 cytokine balance and allergic lung inflammation. Intrauterine undernourished offspring were obtained from dams fed $50 \%$ of the nourished diet of their counterparts and were immunized at 9 weeks of age. We evaluated the cell counts and cytokine protein expression in the bronchoalveolar lavage, mucus production and collagen deposition, and cytokine gene expression and transcription factors in lung tissue 21 days after ovalbumin immunization. Intrauterine undernourishment significantly reduced inflammatory cell airway infiltration, mucus secretion and collagen deposition, in rats immunized and challenged. Intrauterine undernourished rats also exhibited an altered cytokine expression profile, including higher TNF- $\alpha$ and IL-1 $\beta$ expression and lower IL- 6 expression than well-nourished rats following immunization and challenge. Furthermore, the intrauterine undernourished group showed reduced ratios of the IL-4/IFN- $\gamma$ and the transcription factors GATA-3/T-Bet after immunization and challenge. We suggest that the attenuated allergic lung inflammation observed in intrauterine undernourished rats is related to an altered Th1/Th2 cytokine balance resulting from a reduced GATA-3/T-bet ratio.
\end{abstract}




\section{Introduction}

Malnutrition is an important cause of immune suppression and can increase host susceptibility to infectious diseases such as diarrhea and pneumonia by impairing immune responsiveness through a number of different pathways [1]. Cell-mediated and humoral immunity are compromised, leading to thymus and lymph node atrophy, reduced numbers of circulating lymphocytes and dendritic cells [2], increased undifferentiated lymphocyte numbers and depressed serum complement levels $[3,4]$.

There are several critical windows during early life that can increase the risk of chronic adult disease. Failure of the maternal-placental nutrient supply to match fetal demand can result in a range of fetal adaptations and developmental changes. Although these adaptations may be beneficial for short-term survival, they can lead to permanent alterations in body structure and metabolism and thereby to cardiovascular and metabolic diseases during adulthood $[5,6]$.

Several human epidemiological studies have indicated that the incidence of lung function alterations can be associated with birth weight and specifically with maternal malnutrition $[7,8]$. Kitchen et al. [9] concluded that increased bronchial responsiveness is common in school children who were born substantially preterm. Barker et al. [10] found that lower birth weights were associated with decreased lung function as adults and hypothesized that intrauterine conditions causing growth retardation might irreversibly constrain the development of the airways. Additionally, our group has demonstrated that global intrauterine undernourishment leads to significant reductions in lung allergic inflammation in rats [11].

Asthma is a complex syndrome mainly characterized by a variable degree of airflow obstruction, bronchial hyperreactivity and airway inflammation [12]. CD4+ lymphocytes with a T helper 2 (Th2) cytokine phenotype play a pivotal role in the pathogenesis of asthma through orchestrating the activation of the mast cells and eosinophils and releasing cytokines, such as interleukin (IL)-4, IL-5, and IL-13. In contrast, effector Th1 cells are involved in delayed-type hypersensitivity reactions through the production of interferon-gamma (IFN- $\gamma$ ) and IL-2 [13]. IL-4 produced by Th2 cells can block the production of cytokines by Th1 cells, and Th1 cells, by secreting IFN- $\gamma$, can inhibit the allergic eosinophilia and airway hyperresponsiveness [14]; therefore, we hypothesized that intrauterine undernutrition attenuates allergic lung inflammation in rats by altering the balance between Th1 and Th2 cytokine production.

\section{Materials and Methods}

Animals

All procedures conducted in this study were approved and performed in accordance with guidelines established by the ethics committee of the Institute of Biomedical Sciences (041/2001), University of São Paulo. The male and female Wistar rats used were from our colony (Hypertension and Inflammation Laboratory, Institute of Biomedical Sciences, University of São Paulo). They were housed in a $22 \pm 1{ }^{\circ} \mathrm{C}$ environment at $60 \%$ humidity and were maintained on a 12-h light- dark cycle.

\section{Protocol for induction of intrauterine undernutrition}

Timed mating was carried out in age-matched (12- to 16-week-old) female and male Wistar rats. To assess the stage of estrus of the females, vaginal smears were checked before the introduction of the males. Day 1 of the pregnancy was determined as the day on which spermatozoa were detected in the vaginal smear. After confirming mating, the rats were housed individually in standard rat cages. Female rats were randomly divided into two groups: nourished ad libitum and undernourished. Well-nourished female rats were fed a standard commercial rat diet (Nuvital, Nuvital Nutrientes S/A, PR, Brazil) containing protein (minimum 22\%), carbohydrates (maximum 54\%), fat (minimum 4.5\%), cellulose (maximum 8\%), minerals (maximum 10\%), water (maximum 12.5\%), and vitamins. Undernourished female rats were fed the same diet at $50 \%$ of the nourished female rats intake, as determined by the amount of food consumed by the 
control group from day 1 of pregnancy until day 23 (parturition). All rats were fed daily, in the morning, and consumption was determined $24 \mathrm{~h}$ later. After parturition, dams received food ad libitum; therefore, the pups differed only in prenatal dietary experience. To prevent variations in neonatal growth due to the availability of milk during suckling, the litter sizes were standardized to eight pups on day 1 . After suckling, the undernourished dams were sacrificed. The pups were fed with the same diet as the dams after weaning. At 9 weeks of age, the male offspring were used in experiments to evaluate allergic lung inflammation.

\section{Immunization protocol}

Nine-week-old male rats from the well-nourished and undernourished mothers groups were sensitized on days 0 and 7 by intraperitoneal injection of a mixture containing $50 \mu \mathrm{g}$ of ovalbumin (grade III, Sigma, St. Louis, MO, USA) and $1 \mathrm{mg}$ of $\mathrm{Al}(\mathrm{OH})_{3}$ (Rheis, Inc., Berkeley Heights, NJ, USA) in saline (total volume of $0.6 \mathrm{~mL}$ ). At days 14 and 21 after the first immunization, the well-nourished and undernourished offspring were challenged by exposure to an aerosol of ovalbumin generated by an ultrasonic nebulizer (ICEL US800 , SP, Brazil) delivering particles of $0.5-10 \mu \mathrm{m}$ in diameter at approximately $0.75 \mathrm{cc} / \mathrm{min}$ for $20 \mathrm{~min}$. The concentration of ovalbumin in the nebulizer was $2.5 \%$ (wt/vol). These rats constituted the experimental group, and the control group consisted of rats immunized as previously described and challenged with phosphate buffered saline (PBS) solution.

\section{Bronchoalveolar lavage}

The rats were sacrificed by overdose of anesthetic (sodium pentobarbital) $24 \mathrm{~h}$ after exposure to the second aerosol challenge. Bronchoalveolar lavage was collected, and the total and differential cell counts were determined as described previously [15].

\section{Histological analysis}

Lung tissue was harvested, fixed in a $10 \%$ buffered formaline solution and routinely processed for histological inclusion in paraffin. $5 \mu \mathrm{m}$ thick tissue sections were stained with Periodic Acid of Schiff (PAS) to visualize mucus and with Picrosirius to visualize collagen. Morphometrical analysis was performed using a Nikon DXM 1200c digital camera and Nikon NIS - Elements AR 2.30 software. For each stain, the positively staining area $\left(\mu \mathrm{m}^{2}\right)$ was measured for 10 bronchioles per slide, or the maximum number of bronchioles in each slide, for 7 rats per group.

\section{Flow cytometric analyses of lymphocytes}

Lymphocyte subpopulations phenotypes were assessed by three color FACS, using a FACScalibur Cytometer equipped with Cell Quest software (Becton and Dickinson, San Jose, CA, USA) using gates defined by forward and side scatter properties. BAL cells were incubated with Fluorescein Isothiocyanate (FITC) or R-Phycoerythrin (PE) labeled monoclonal antibodies directed against $\mathrm{CD}_{4}$ (clone $\mathrm{H} 129.19$ ) and $\gamma \delta \mathrm{T}$ (clone GL3) and adjusted to a concentration of $1 \times 10^{5}$ cells $/ \mathrm{ml}$ in PBS supplemented with $5 \%$ fetal bovine serum and sodium azide $(0.1 \%)$.

\section{Quantification of cytokines in the bronchoalveolar lavage}

A bioplex (Millipore - Billerica, MA, USA) rat cytokine assay kit in conjunction with the Bioplex 200 Suspension Array System/Luminex (Bio-Rad, Hercules, CA, USA) was used to measure IL-6 and tumor necrosis factor (TNF)- $\alpha$ in the bronchoalveolar lavage supernatant. The kit was used according to the manufacturer's instructions. The data were analyzed using BioPlex Manager software version 4.0. The standard curves used ranged from 1.95 to $32,000 \mathrm{pg} / \mathrm{ml}$. The lower limit of detection for each cytokine was $5.56 \mathrm{pg} / \mathrm{ml}$ for IL- 6 and $3.36 \mathrm{pg} / \mathrm{ml}$ for TNF- $\alpha$.

\section{Real Time PCR}

Lung samples, including bronchi, bronchioles and parenchyma, were quickly frozen in liquid nitrogen. Total RNA was isolated from the lung tissue using the TRizol Reagent (Invitrogen, USA), and the RNA concentration and the purity of the samples were determined by spectrophotometer readings at 260 $\mathrm{nm}$ and $280 \mathrm{~nm}$. First-strand cDNA was synthesized using MML-V reverse transcriptase (Promega, USA). Reverse transcriptase polymerase chain reaction was performed using the following TaqMan PCR assays: IL-1 $\beta$ (Rn00580432_m1), IL-4 (Rn01456866_m1), TNF- $\alpha$ (Rn99999017_m1) and IFN- $\gamma$ (Rn00594078_m1), and the housekeeper gene hypoxanthine guanine phosphoribosyltransferase (HPRT) (Rn01527838_g1) (Applied Biosystem, California, USA). Real-time PCR was performed to assess GATA-3 and T-bet expression 
Table 1. Oligonucleotides sequence

\begin{tabular}{lll}
\hline Gene & Forward & Reverse \\
\hline GATA-3 & 5'-GCC TGT GCA AAA GAG ATT TCA GAT-3' & 5'-TGA TTC ACA GAG CAT GTA GGC C-3' \\
T-bet & 5'-CCA GTA TCC TGT TCC CAG CC-3' & 5'-CCA GTA TCC TGT TCC CAG CC-3' \\
HPRT & 5'-CTC ATG GAC TGA TTA TGG ACA GGA C-3' & 5'-GCA GGT CAG CAA AGA ACT TAT AGC C-3' \\
\hline
\end{tabular}

Table 2. Effect of intrauterine malnutrition on the cells in the bronchoalveolar lavage

\begin{tabular}{llll}
\hline & Total cells $\left(\times 10^{4} / \mathrm{mL}\right)$ & Eosinophils $\left(\times 10^{4} / \mathrm{mL}\right)$ & Neutrophils $\left(\times 10^{4} / \mathrm{mL}\right)$ \\
\hline NR OVA/PBS & $50.0 \pm 6.7$ & $0.27 \pm 0.1$ & $2.07 \pm 1.0$ \\
NR OVA/OVA & $255.5^{*} \pm 53.6$ & $129.5^{*} \pm 30.2$ & $13.89^{*} \pm 4.9$ \\
UR OVA/PBS & $55.5 \pm 10.9$ & $0.31 \pm 0.18$ & $1.69 \pm 1.1$ \\
UR OVA/OVA & $168.5^{* * \#} \pm 23.3$ & $72.89^{* * *} \pm 16.9$ & $9.69^{* *} \pm 2.9$
\end{tabular}

Values indicate mean \pm SEM, $n=7$. NR OVA/PBS = well-nourished group immunized with ovalbumin and challenged with phosphate buffered saline; NR OVA/OVA = well-nourished group immunized and challenged with ovalbumin; UR OVA/PBS = intrauterine undernourished group immunized with ovalbumin and challenged with phosphate buffered saline; UR OVA/OVA = intrauterine undernourished group immunized and challenged with ovalbumin; *different from NR OVA/PBS; "different from UR OVA/PBS; "different from NR OVA/OVA. $P<0.05$.

using a SYBR Green assay (Applied Biosystem), in conjunction with specific SYBR Green HPRT analysis. The sequences of oligonucleotides used are depicted in in Table 1. The cycling conditions were as follows: $10 \mathrm{~min}$ at $95{ }^{\circ} \mathrm{C}$, then 45 cycles of $15 \mathrm{~s}$ at $95^{\circ} \mathrm{C}$ and $1 \mathrm{~min}$ at $60^{\circ} \mathrm{C}$. The amount of the target gene was normalized first to an endogenous reference (HPRT) and then relative to a calibrator, the sample with the lowest expression, using the $2-\Delta \Delta$ Ct method. Thus, steady-state mRNA levels were expressed as an $\mathrm{n}$-fold difference relative to the calibrator. Analyses were performed with Sequence Detection Software 1.9 (SDS).

Serum total protein and albumin assay

For serum protein analysis, the rats were anesthetized (sodium pentobarbital, $45 \mathrm{mg} / \mathrm{kg}$, intraperitoneally; Cristalia, São Paulo, Brazil) and blood was collected from the abdominal aorta. The total protein level was determined by the method described by Bradford [16], using bovine albumin as a standard. A kit was used to determine serum albumin concentration (Biobrás, São Paulo, Brazil).

\section{Statistical analysis}

Data are expressed as the means \pm SEM. Statistical evaluation of the data was conducted using a twoway analysis of variance followed by the Tukey-Kramer multiple comparison test. Student's $t$ test was used when necessary. A $P$ value lower than 0.05 was considered statistically significant.

\section{Results}

\section{Offspring characteristics}

Body weight. Maternal undernutrition resulted in fetal growth retardation, as evidenced by a marked reduction in the birth weight of rats offspring exposed to intrauterine undernutrition compared to the well-nourished group $3.8 \pm 0.1$ versus $5.6 \pm 0.1 \mathrm{~g}$, respectively, $n=22, P<0.001)$. At 9 weeks of age, undernourished male offspring had a body weight (258.3 $\pm 5.7 \mathrm{~g})$ similar to that of well-nourished male offspring $(260.3 \pm 4.2 \mathrm{~g})$.

Nutritional state - serum total protein and albumin assay. No difference in the nutritional state was observed between the well-nourished and the intrauterine undernourished offspring. The well-nourished and intrauterine undernourished groups did not differ in total protein levels ( $51.85 \pm 1.9$ versus $51.37 \pm 2.9 \mu \mathrm{g} / \mu \mathrm{L}$, respectively) or serum albumin $(2.59 \pm$ 0.19 versus $2.41 \pm 0.15 \mu \mathrm{g} / \mu \mathrm{L}$, respectively). 


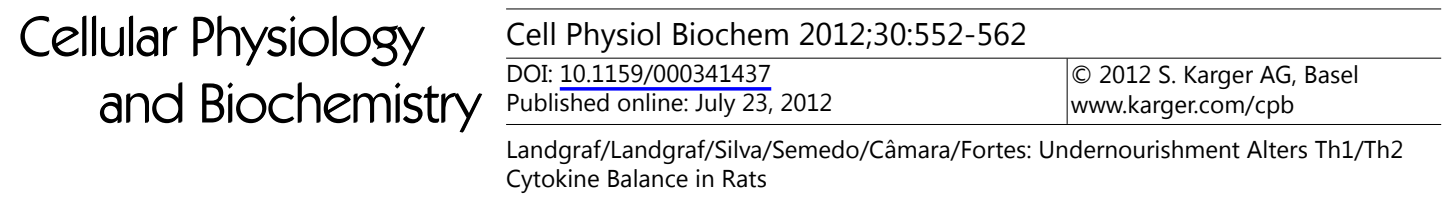

Fig. 1. Effect of intrauterine malnutrition on the bronchoalveolar lavage lymphocytes subpopulations. Rats were immunized through i.p. injection of ovalbumin/alumen with a booster injection 7 days later. Rats were challenged twice with aerosolized ovalbumin (2.5\%, $20 \mathrm{~min})$ on days 14 and 21 postimmunization. Bronchoalveolar lavage was performed $24 \mathrm{~h}$ after the second challenge. The cells were incubated with fluorochromelabeled monoclonal antibodies to $\mathrm{CD}_{4}$ and $\gamma \delta \mathrm{T}$ cells and analyzed by FACS. NR OVA/PBS = wellnourished group immunized with ovalbumin and challenged with phosphate buffered saline; NR OVA/OVA = well-nourished group immunized and challenged with ovalbumin; UR OVA/PBS = intrauterine undernourished group immunized with ovalbumin and challenged with phosphate buffered saline; UR OVA/OVA = intrauterine undernourished group immunized and challenged with ovalbumin. The results are presented as the mean \pm SEM. $n=6 ;{ }^{*} \mathrm{P}<0.05$.

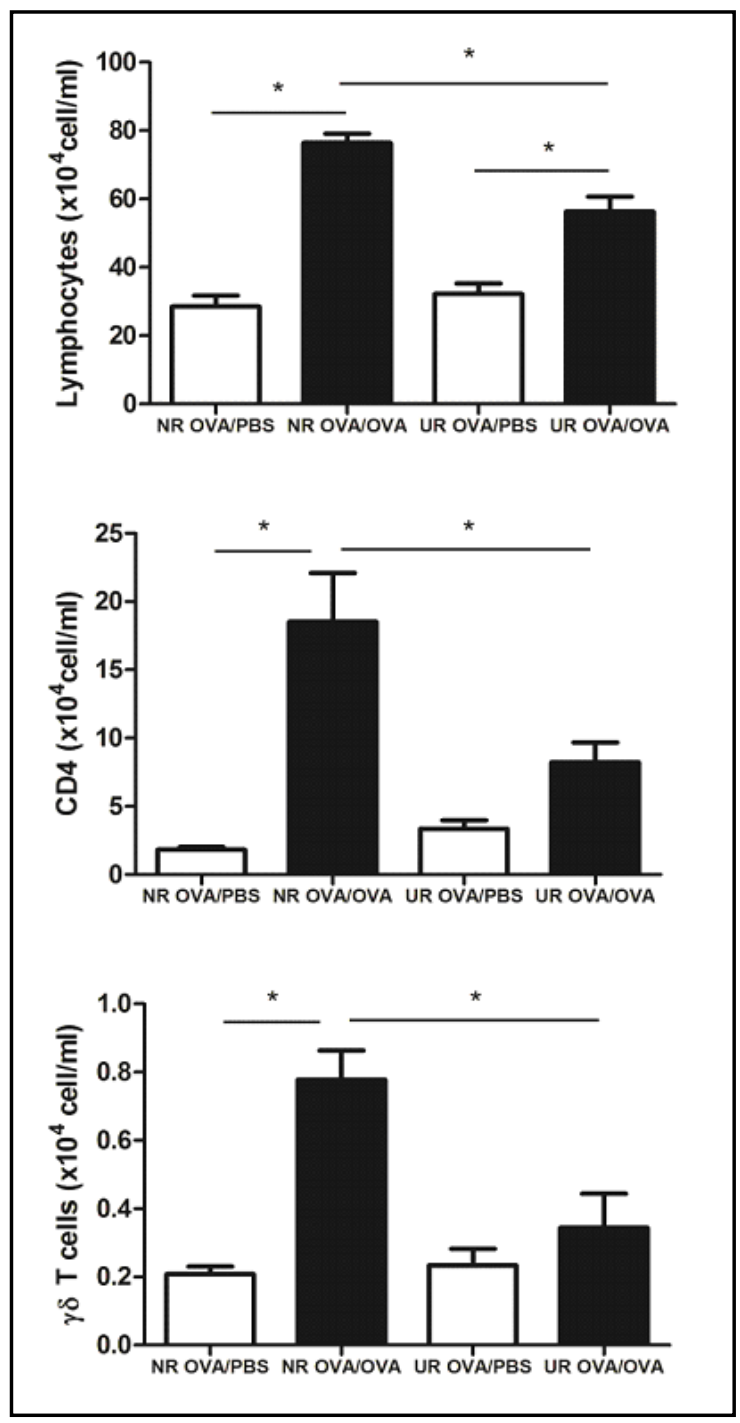

Effect of intrauterine undernutrition on allergic lung inflammation

Cells in bronchoalveolar lavage. Eosinophils and neutrophils: In both undernourished and well-nourished rats, immunization and challenge with ovalbumin increased total cell infiltration in the bronchoalveolar lavage, indicating that both groups responded to the immunological stimuli. The increased cell numbers mainly reflected enhanced eosinophil and neutrophil recruitment to the lung. The increases in total leukocytes and eosinophils following immunization and challenge were blunted in the undernourished rats compared to the well-nourished rats (Table 2).

Lymphocytes: There were significant increases in the numbers of lymphocytes in both the immunized and challenged groups compared to the respective control groups. Immunized and challenged well-nourished rats also exhibited a significant increase in CD4 and $\gamma \delta \mathrm{T}$ cells compared to their control group. In contrast, immunization and challenge with ovalbumin did not induce a significant CD4 and $\gamma \delta$ T cells infiltration in the undernourished group (Fig.1).

\section{Histopathologic analysis}

Collagen deposition and mucus production. Although immunization and challenge with ovalbumin induced significant increases in collagen deposition and mucus production in both well-nourished and intrauterine undernourished groups, these were both diminished 


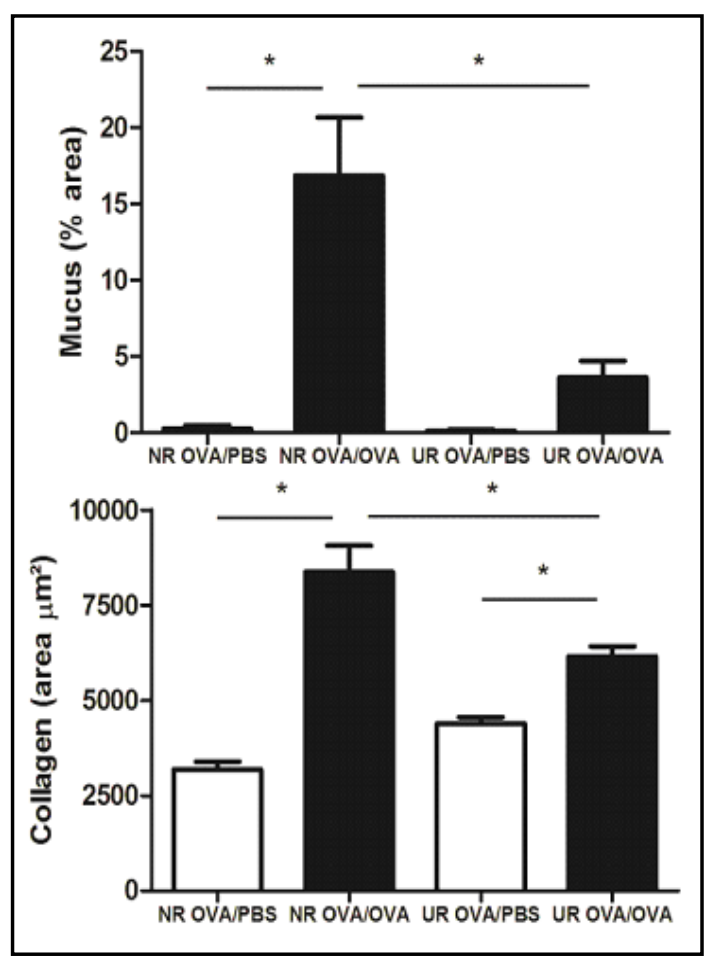

Fig. 2. Histopathologic analysis of collagen and mucus production. Lungs were sliced into $5-\mu \mathrm{m}$ sections and stained with periodic acid-Schiff and hematoxylin to evaluate mucus-producing cells or with picrosirius to evaluate collagen fibers. Quantitative digital morphometric analysis was performed using Metamorph 6.0 (Universal Images Corporation, USA). Bronchi circumference and the PAS stained areas were electronically measured, and the mucus index was determined by the following formula: (PAS stained area / bronchial circumference area) $\times 100$. NR OVA/PBS $=$ well-nourished group immunized with ovalbumin and challenged with phosphate buffered saline; NR OVA/OVA = well-nourished group immunized and challenged with ovalbumin; UR OVA/PBS $=$ intrauterine undernourished group immunized with ovalbumin and challenged with phosphate buffered saline; UR OVA/OVA = intrauterine undernourished group immunized and challenged with ovalbumin. The results are presented as the mean \pm SEM. $\mathrm{n}=6-8{ }^{*} \mathrm{P}<0.05$.

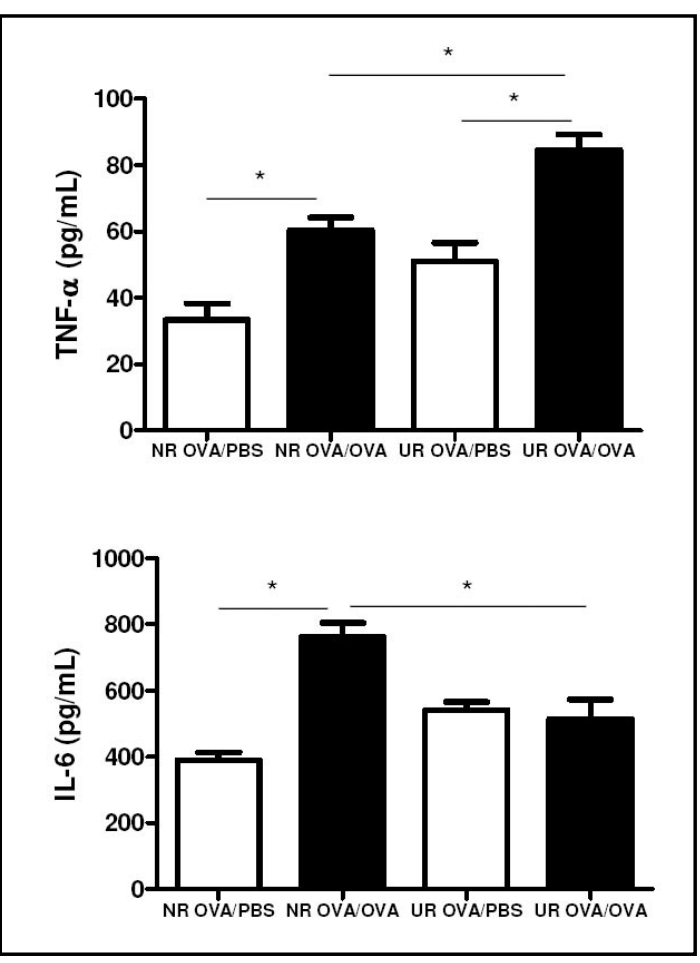

Fig. 3. Cytokines in bronchoalveolar lavage: Rats were immunized by i.p. injection of ovalbumin/ alumen with one booster injection 7 days later. Rats were challenged twice with aerosolized ovalbumin $(2.5 \%, 20 \mathrm{~min})$ on days 14 and 21 post-immunization. Bronchoalveolar lavage was performed $24 \mathrm{~h}$ after the second challenge. Tumor necrosis factor-alpha (TNF- $\alpha$ ) and Interleukin (IL)-6 concentrations in the BAL $(\mathrm{pg} / \mathrm{ml})$ were quantified by Bioplex, as described in "Materials and Methods". NR OVA/PBS = well-nourished group immunized with ovalbumin and challenged with phosphate buffered saline; NR OVA/OVA = well-nourished group immunized and challenged with ovalbumin; UR OVA/PBS = intrauterine undernourished group immunized with ovalbumin and challenged with phosphate buffered saline; UR OVA/OVA = intrauterine undernourished group immunized and challenged with ovalbumin. The results are presented as the mean \pm SEM. $n=6$; ${ }^{*} \mathrm{P}<0.05$.

in the undernourished group compared to the well-nourished group (Fig. 2).

\section{Cytokine assays}

Cytokine measurement in the bronchoalveolar lavage. In contrast to the well-nourished group, immunization and challenge with ovalbumin did not alter IL-6 production in the undernourished group. However, immunization and challenge with ovalbumin evoked a significant increase in the TNF- $\alpha$ production in the intrauterine undernourished group compared with the well-nourished group (Fig. 3).

Cytokine gene expression in lung tissue. The levels of IL- $1 \beta$, TNF- $\alpha$ and IFN- $\gamma$ expression were similar in the well-nourished and undernourished groups following challenge 


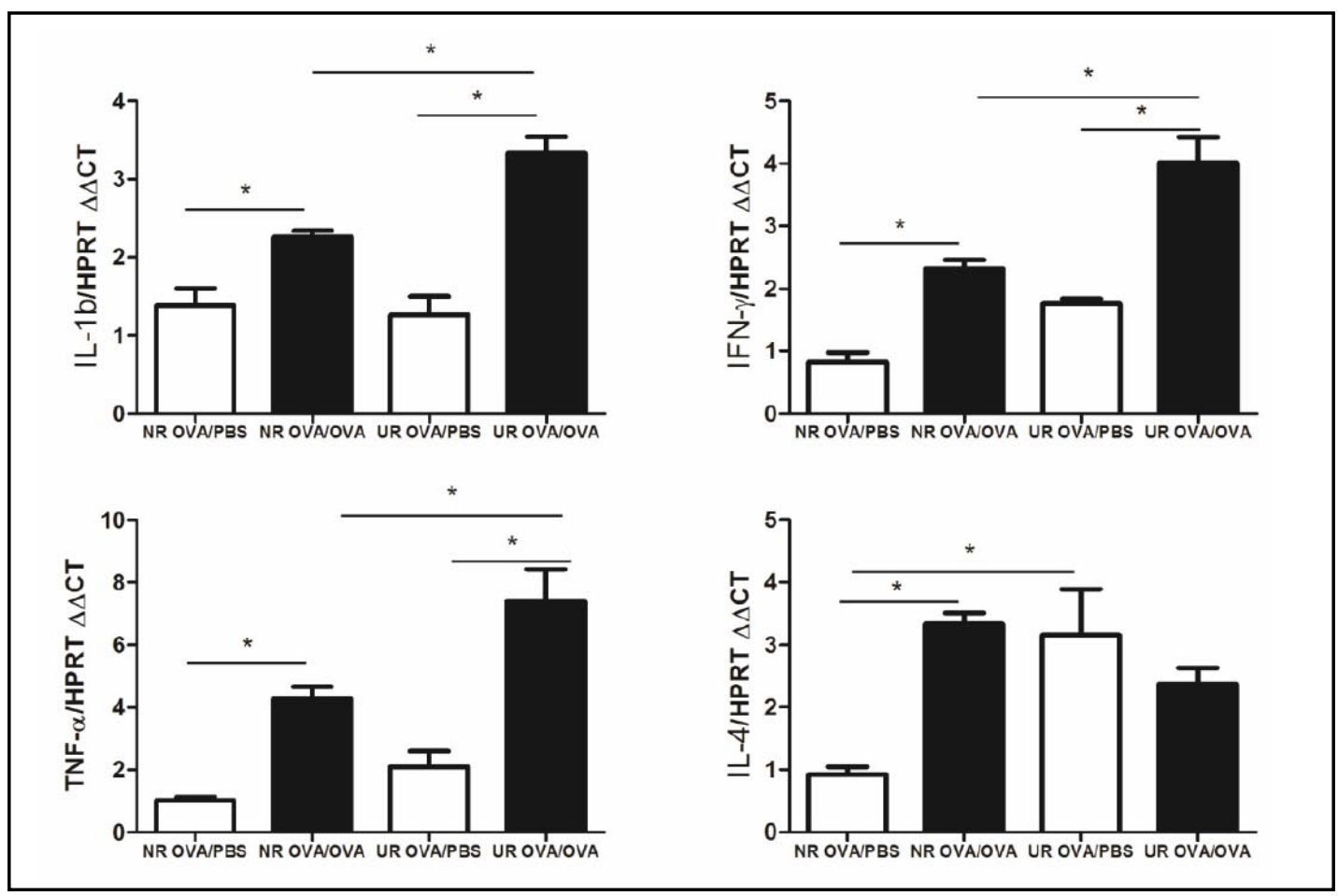

Fig. 4. Expression of cytokines and transcription factors in lung tissue: Interleukin-1 beta (IL-1b), interferon-gamma (IFN- $\gamma$ ), tumor necrosis factor-alpha (TNF- $\alpha$ ), interleukin-6 (IL-6) and interleukin-4 (IL-4) messenger RNA (mRNA) expression was evaluated by real-time polymerase chain reaction (PCR). Samples were normalized based on the endogenous housekeeping gene hypoxanthine-guanine phosphori bosyltransferase (HPRT). NR OVA/PBS = well-nourished group immunized with ovalbumin and challenged with phosphate buffered saline; NR OVA/OVA = well-nourished group immunized and challenged with ovalbumin; UR OVA/PBS = intrauterine undernourished group immunized with ovalbumin and challenged with phosphate buffered saline; UR OVA/OVA = intrauterine undernourished group immunized and challenged with ovalbumin. The results are presented as the mean \pm SEM. $n=6$; ${ }^{*} \mathrm{P}<0.05$.

with PBS. Although expression of these cytokines was enhanced by immunization and challenge with ovalbumin in both groups, TNF- $\alpha$, IL- $1 \beta$ and IFN- $\gamma$ expression was higher in undernourished rats than in well-nourished rats. IL-4 expression was significantly increased in undernourished rats challenged with PBS compared to rats from the well-nourished group challenged with PBS. In contrast to undernourished rats, immunization and challenge with ovalbumin increased the IL-4 expression in well-nourished rats (Fig. 4).

\section{IL-4/IFN- $\gamma$ ratio and GATA-3/T-bet ratio in lung tissue}

To evaluate the balance between the Th1 and Th2 cytokine responses, we calculated the ratio between IFN- $\gamma$, a marker for the Th1 response, and IL-4, a marker for the Th2 response. We observed an enhanced IL-4/IFN- $\gamma$ ratio in the undernourished group challenged with PBS compared to the well-nourished group challenged with PBS. Unlike the well-nourished rats, immunization and challenge with ovalbumin caused a reduction in the IL-4/IFN- $\gamma$ ratio in undernourished rats (Fig. 5A). We found that immunization and challenge with ovalbumin increased the GATA-3/T-bet ratio in well-nourished rats (Fig. 5B), consistent with the allergic response developed by this group. Rats from the undernourished group challenged with PBS showed a higher GATA-3/T-bet ratio than those from the respective well-nourished group. After immunization and challenge with ovalbumin, the GATA-3/T-bet ratio decreased significantly in the undernourished group. This result may reflect the attenuated lung allergic inflammation observed in this group (Fig. 5B). 
Fig. 5. A: Ratio of IL-4/IFN- $\gamma$ : mRNA levels of interleukin-4 (IL-4) and interferon-gamma (IFN- $\gamma$ ) were evaluated by real-time polymerase chain reaction (PCR). Samples were normalized to the endogenous gene hypoxanthine-guanine phosphoribosyltransf erase (HPRT). Data are presented as the ratio of the expression of the IL-4 gene to the expression of the IFN$\gamma$ gene. $n=6$; ${ }^{*} \mathrm{P}<0.05$. B: Ratio of GATA-3/T-bet: mRNA levels of GATA-3 and T-bet were evaluated by realtime polymerase chain reaction (PCR). Samples were normalized to the endogenous gene hypoxanthineguanine phosphoribosyltransferase (HPRT). Data are presented as the ratio of the expression of the GATA-3 gene to the expression of the T-bet gene. NR OVA/PBS = well-nourished group immunized with ovalbumin and challenged with phosphate buffered saline; NR OVA/OVA = well-nourished group immunized and challenged with ovalbumin; UR OVA/PBS = intrauterine undernourished group immunized with ovalbumin and challenged with phosphate buffered saline; UR OVA/OVA = intrauterine undernourished group immunized and challenged with ovalbumin. $\mathrm{n}$ $=6 ;{ }^{*} \mathrm{P}<0.05$.

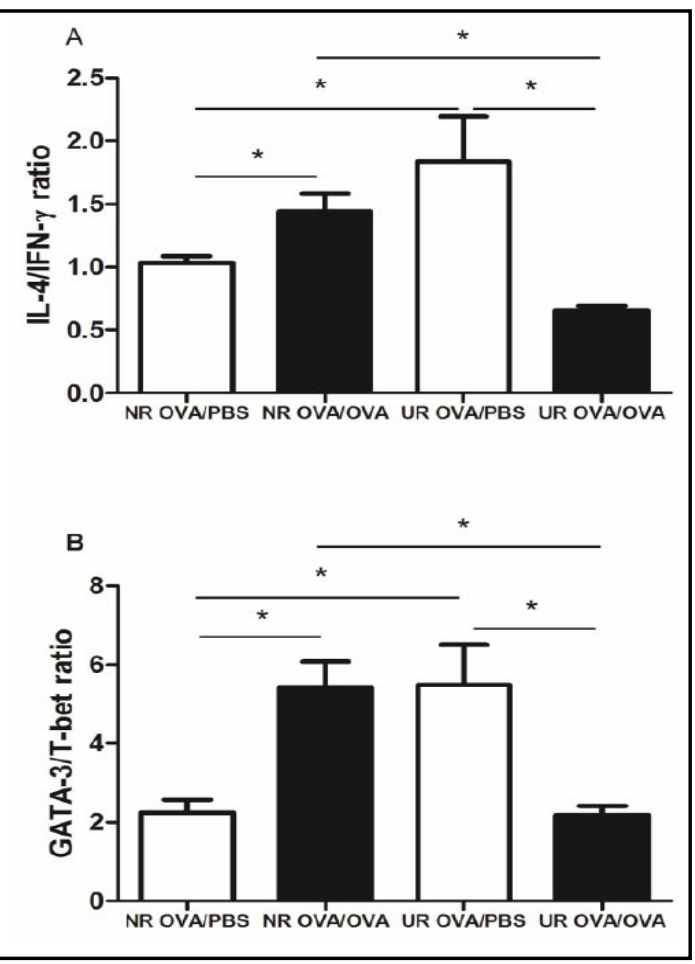

\section{Discussion}

In this work, we demonstrated that the allergic inflammatory response is attenuated in intrauterine undernourished rats. This attenuation is associated with a reduced GATA$3 /$ T-bet ratio and an imbalance in Th1/Th2 cytokines, which leads to a shift in the Th1/Th2 balance towards Th1.

Maternal food restriction during the entirety of pregnancy caused a significant reduction in the offspring birth weight and fulfilled our criteria for this study. We ruled out the current nutritional status of rats as the cause of the observed changes because no difference was observed in biochemical parameters such as serum albumin and total protein levels between well-nourished and undernourished rats challenged with either PBS or ovalbumin.

After immunization and challenge, both well-nourished and undernourished offspring groups showed a significant influx of total cells, eosinophils and neutrophils in BAL, indicating the efficacy of our protocol.

$\mathrm{CD}_{4}^{+}$and $\gamma \delta \mathrm{T}$ cells play an important role on the development of allergic lung disease. $\gamma \delta \mathrm{T}$ cells are required to induce allergen-specific IgE and IgG1 responses and Th2-mediated airway inflammation in a mouse model of asthma. [17]. $\mathrm{CD}_{4}{ }^{+} \mathrm{T}$ cells secrete $\mathrm{Th} 2$ cytokines that can contribute to many of the pathophysiological features of asthma that perpetuate the disease, including eosinophil accumulation in airways and mucus secretion [18]. Increased numbers of activated T cells and eosinophils infiltrating the lung in asthma patients typically correlate with the severity of the disease [19]. Because cell migration depends on the availability of circulating cells, we hypothesize that the reduced influx of $\mathrm{CD}_{4}^{+}$and $\gamma \delta \mathrm{T}$ cells and eosinophils into the lung in undernourished rats challenged with ovalbumin could be related to the hypocellularity in the bone marrow and the peripheral blood observed in this group, as demonstrated in our previous study [20].

Other cardinal features of chronic lung inflammation include excessive mucus production and collagen deposition. Excessive mucus production can lead to physical obstruction of the smaller caliber airways. In addition to enhanced mucus production, the decline in lung function observed in asthmatics is the result of airway remodeling characterized by significant subepithelial deposition of extracellular matrix molecules, including collagens [21, 22]. IL-6 
is a key regulator of the specific hallmark features of allergic airway inflammation, and IL-6 deficient mice present attenuated mucus production [22] and fibrotic changes [21] during allergic airway inflammation. This relationship was observed in our study, as the increased mucus production and collagen deposition in well-nourished rats challenged by ovalbumin was accompanied by elevated IL-6 production. Additionally, undernourished rats challenged with ovalbumin exhibited reduced mucus production and collagen deposition in conjunction with lowered IL-6 levels. As excessive mucus production and collagen accumulation is correlated with the severity of allergic lung inflammation in humans and experimental animal models $[21,22]$, the lack of an increase in IL-6 levels associated with low mucus secretion and collagen deposition could contribute to the diminished intensity of allergic lung inflammation in undernourished rats.

Naive $\mathrm{CD}_{4}^{+} \mathrm{Th} 0$ cells are bipotential, and they are considered precursors of both Th1 and Th2 cells. Th1 cells primarily secrete IFN- $\gamma$, IL-2, and TNF- $\alpha$ and Th2 cells secrete primarily IL-4, IL-10, and IL-13. Th1 and Th2 responses are mutually inhibitory, with IL-12 and IFN- $\gamma$ inhibiting the activity of Th2 cells and IL- 4 and IL-10 inhibiting the activity of Th1 cells [23, 24]

IL-4 plays a key role in the development of allergic inflammation and asthma through inducing the IgE isotype switching [25] and eosinophil infiltration [26]. Unexpectedly, we found that basal expression of IL-4 in the undernourished group was very high, but despite this immunization and challenge did not increase IL-4 expression. As experimental IL-4 neutralization prevents the development of allergen-specific IgE and reduces eosinophilic inflammation [27], the failure in increasing IL-4 levels after immunization and challenge might be another factor contributing to the impairment of allergic lung inflammation in the undernourished group.-

TNF- $\alpha$ is a Th1 cytokine that has been implicated in asthmatic airway inflammation [28]. During asthma exacerbations, enhanced TNF- $\alpha$ levels are associated with increased ICAM-1, VCAM-1, and E-selectin expression and a consequent influx of inflammatory cells into the lung [29]. Despite elevated TNF- $\alpha$ expression observed in the undernourished rats following ovalbumin challenge, the influx of eosinophils, neutrophils and lymphocytes was lower than in the well-nourished group. The hypocellularity and the reduced expression of adhesion molecules that we previously observed in undernourished rats $[20,30]$ may have contributed to these results.

IFN- $\gamma$ production is believed to be restricted to activated natural killer (NK) cells, $\mathrm{CD}_{4}^{+}$ Th1 cells, and $\mathrm{CD}_{8}{ }^{+} \mathrm{T}$ cytotoxic cells [31]. However, several studies have identified additional IFN- $\gamma$-secreting cell types, including $\gamma \delta \mathrm{T}$ cells, NKT cells, macrophages, dendritic cells, naive $\mathrm{CD}_{4}^{+} \mathrm{T}$ cells, and even B cells [31,32]. We note that, despite a significant reduction in $\mathrm{CD}_{4}^{+} \mathrm{T}$ and $\gamma \delta \mathrm{T}$ cells in undernourished rats immunized and challenged with ovalbumin, IFN- $\gamma$ production was higher in this group than in the well-nourished rats immunized and challenged with ovalbumin. Therefore, we speculate that these cells might be more activated, with enhanced IFN- $\gamma$ production despite their reduced numbers. However, further studies are necessary to test this hypothesis.

In addition to the alterations IL-4 and IFN- $\gamma$ expression seen in the undernourished group, we observed a lower IL-4/ IFN- $\gamma$ ratio in this group compared to the well-nourished group following immunization and challenge with ovalbumin. IL-4 is a known Th2 immune response marker and IFN- $\gamma$ is a Th1 immune response marker, and these cytokines are mutually cross regulatory [14]; therefore, we suggest that intrauterine undernourishment could lead to a reduced IL-4/IFN- $\gamma$ ratio and a consequent imbalance between Th1 and Th2 cytokines that compromises allergic lung inflammation development in rats.

The mechanisms by which intrauterine malnutrition alter the expression of cytokines are not known, but hormones such as glucocorticoids may be involved. We previously demonstrated that intrauterine undernourished rats have high corticosterone levels, and we suggested that this hormone contributed to attenuated lung allergic inflammation observed in this group [11]. Prednisolone treatment in asthma has been shown to modulate cytokine expression, decreasing IL-4 levels and increasing IFN- $\gamma$ levels and consequently inhibiting 
local bronchial inflammatory infiltration and tissue eosinophilia [33]. This led us to suggest that, following intrauterine undernourishment, high corticosterone levels contribute to increased IFN- $\gamma$ level and impair the IL-4 expression, affecting the development of the pulmonary allergic inflammation in intrauterine undernourished rats.

The development of Th1 and Th2 cells has been shown to be primarily regulated by the expression of transcription factors. GATA-3 is indispensable for directing Th2 differentiation, and it plays an important role in inducing the expression of Th2 cytokines [34]. IFN- $\gamma$ activates T-bet, a member of the T-box family of transcription factors, resulting in the expression of genes encoding Th1 cytokines and suppressing genes encoding Th2 cytokines $[35,36]$. The ratio between the essential transcription factors GATA-3 and T-bet has been reported to reflect the Th2 and Th1 cytokine profile in mixed cell populations [37]. In our study, a higher GATA-3/T-bet ratio was observed in the undernourished group than in the well-nourished group both following challenged with PBS. Furthermore, a reduction in GATA-3/T-bet ratio was observed in undernourished rats following the induction of allergic pulmonary inflammation compared to well-nourished rats. T-bet may negatively regulate GATA-3 expression [38], and inhibiting GATA-3 suppresses Th2 cytokines and allergic airway inflammation [39]; therefore, a Th1 shift induced by T-bet overexpression could reduce the GATA-3/T-bet ratio in the undernourished group during allergic pulmonary inflammation.

In summary, we propose that the attenuated allergic lung inflammation observed in intrauterine undernourished rats is closely linked to their altered Th1/Th2 cytokine balance leading to a shift in the Th1/Th2 balance towards Th1 and that this imbalance is a consequence of their reduced GATA-3/T-bet ratio during allergic lung inflammation.

\section{Acknowledgements}

We would like to acknowledge Paulo Bernardo Albe for his technical assistance. This study was supported by the Conselho Nacional de Desenvolvimento Científico e Tecnológico (CNPq), Fundo de Auxílio aos Docentes e Alunos (FADA-UNIFESP) and the Fundação de Amparo à Pesquisa do Estado de São Paulo (07/07139-3, 09/09849-3, 09/52119-6, 10/01404-0, 12/02270-2).

\section{References}

1 Jones KDJ, Berkley JA, Warner JO: Perinatal nutrition and immunity to infection. Pediatr Allergy Immunol 2010;21:564-576.

2 Cunha WDS, Friedler G, Vaisberg M, Egami MI, Costa Rosa LFB: Immunosuppression in undernourished rats: the effect of glutamine supplementation. Clin Nutr 2003;22:453-457.

-3 Schaible UE, Kaufmann SHE: Malnutrition and infection: complex mechanisms and global impacts. PLoS Med 2007;4:e115.

-4 Nájera 0, González C, Toledo G, López L, Ortiz R: Flow cytometry study of lymphocyte subsets in malnourished and well-nourished children with bacterial infections. Clin Diagn Lab Immunol 2004;11:577-580.

5 Barker DJ, Osmond C, Simmonds SJ, Wield GA: The relation of small head circumference and thinness at birth to death from cardiovascular disease in adult life. Brit Med J 1993;306:422-426.

6 Godfrey KM, Barker DJ: Fetal nutrition and adult disease. Am J Clin Nutr 2000;71:1344s-1352s.

7 Langley-Evans SC, Phillips GJ, Jackson AA: Fetal exposure to low protein maternal diet alters the susceptibility of young adult rats to sulfur dioxide-induced lung injury. J Nutr 1997;27:202-209.

-8 Edwards CA, Osman LM, Godden DJ, Campbell DM, Douglas JG: Relationship between birth weight and adult lung function: controlling for factors. Thorax 2003;58:1061-1065.

-9 Kitchen WH, Olinsky A, Doyle LW, Ford GW, Murton LJ, Slonim L, Callanan C: Respiratory health and lung function in 8-year old children of very low birth weight: a cohort study. Pediatrics 1992;89:1151-1158.

10 Barker DJP, Godfrey KM, Fall C, Osmond C,Winter PD, Shaheen SO: Relation of birth weight and childhood respiratory infection to adult lung function and death from chronic obstructive airways disease. BMJ 1991;303:671-675. 
Landgraf/Landgraf/Silva/Semedo/Câmara/Fortes: Undernourishment Alters Th1/Th2

Cytokine Balance in Rats

11 Landgraf MA, Landgraf RG, Jancar S, Fortes ZB: Influence of age on the development of immunological lung response in intrauterine undernourishment. Nutrition 2008;24:262-269.

12 Kay AB: The role of eosinophils in the pathogenesis of asthma. Trends Mol Med 2005;11:148-152.

13 Mazzarella G, Bianco A, Catena E, De Palma R, Abbate GF: Th1/Th2 lymphocyte polarization in asthma. Allergy 2000;61:6-9.

14 Chung KF, Barnes PJ: Cytokines in asthma. Thorax 1999;54:825-857.

15 Gama Landgraf R, Sirois P, Jancar S: Differential modulation of murine lung inflammation by bradykinin B1 and B2 selective receptor antagonists. Eur J Pharmacol 2003;460:75-83.

16 Bradford MM: A rapid and sensitive method for the quantitation of microgram quantities of protein utilizing the principle of protein-dye binding. Anal Biochem 1976;72:248-254.

$\checkmark 17$ Zuany-Amorim C, Ruffié C, Hailé S, Vargaftig BB, Pereira P, Pretolani M: Requirement for gamma delta T cells in allergic airway inflammation. Science 1998;280:1265-1267.

18 Umetsu D, DeKruyff RH: Th1 and Th2 $\mathrm{CD}_{4}^{+}$cells in human allergic diseases. J Allergy Clin Immunol 1997;100:1-6.

19 Burchell JT, Wikstrom ME, Stumbles PA, Sly PD, Turner DJ: Attenuation of allergen-induced airway hyperresponsiveness is mediated by airway regulatory T cells. Am J Physiol Lung Cell Mol Physiol 2009;296:L307-319.

20 Landgraf MA, Tostes Rde C, Borelli P, Zorn TM, Nigro D, Carvalho MH, Fortes ZB: Mechanisms involved in the reduced leukocyte migration in intrauterine undernourishment. Nutrition 2007;23:145-156.

21 Qiu Z, Fujimura M, Kurashima K, Nakao S, Mukaida N: Enhanced airway inflammation and decreased subepithelial fibrosis in IL-6-deficient mice following chronic exposure to aerosolized antigen. Clin Exp Allergy 2004;34:1321-1328.

-22 Neveu WA, Allard JB, Dienz O, Wargo MJ, Ciliberto G, Wittaker LA, Rincon M: IL-6 is required for airway mucus production induced by inhaled fungal allergens. J Immunol 2009; 183:1732-1738.

23 Mosmann TR, Sad S: The expanding universe of T-cell subsets: Th1, Th2 and more. Immunol Today 1996;17:138-146.

24 Diehl S, Rincón M: The two faces of IL-6 on Th1/Th2 differentiation. Mol Immunology 2002;39:531-536. Paul WE: Interleukin-4: a prototypic immunoregulatory lymphokine. Blood 1991;77:1859-1870

26 Schleimer RP, Sterbinsky SA, Kaiser J, Bickel CA, Klunk DA, Tomioka K, Newman W, Luscinskas FW, Gimbrone MA Jr, McIntyre BW, Bochner BS: IL-4 induces adherence of human eosinophils and basophils but not neutrophils to endothelium: association with expression of VCAM-1. J Immunol 1992;148:10861092.

-27 Sugita M, Kuribayashi K, Nakagomi T, Miyata S, Matsuyama T, Kitada O: Allergic bronchial asthma: airway inflammation and hyperresponsiveness. Intern Med 2003;42:636-643.

28 Berry M A, Hargadon B, Shelley M, Parker D, Shaw DE, Green RH, Bradding P, Brightling CE, Wardlaw AJ, Pavord ID: Evidence of a role of tumor necrosis factor $\alpha$ in refractory asthma. N Engl J Med 2006;354:697708

29 Kips JC, Tavernier JH, Joos GF, Peleman RA, Pauwels RA: The potential role of tumor necrosis factor a in asthma. Clin Exp Allergy 1993;23:247-250.

-30 Landgraf MA, Martinez LL, Rastelli VM, Franco Mdo C, Soto-Suazo M, Tostes Rde C, Carvalho MH, Nigro D, Fortes ZB: Intrauterine undernutrition in rats interferes with leukocyte migration, decreasing adhesion molecule expression in leukocytes and endothelial cells. J Nutr 2005;135:1480-1485.

-31 Boehm U, Klamp T, Groot M, Howard JC: Cellular responses to interferon-gamma. Annu Rev Immunol 1997;15:749-795.

32 Frucht DM, Fukao T, Bogdan C, Schindler H, O‘Shea JJ, Koyasu S: IFN-g production by antigen-presenting cells: mechanisms emerge. Trends Immunol 2001;22:556-560.

-33 Bentley AM, Hamid Q Robinson DS, Schotman E, Meng Q, Assoufi B, Kay AB, Durham SR: Prednisolone treatment in asthma. Am J Resp Crit Care Med 1996;153:551-556.

34 Usui T, Preiss JC, Kanno Y, Yao ZJ, Bream JH, O'Shea JJ, Strober W: T-bet regulates Th1 responses through essential effects on GATA-3 function rather than on IFNG gene acetylation and transcription. J Exp Med 2006,203:755-766.

-35 Szabo SJ, Kim ST, Costa GL, Zhang X, Fathman CG, Glimcher LH: A novel transcription factor, T-bet, directs Th1 lineage commitment. Cell 2000;100:655-669.

-36 Szabo SJ, Sullivan BM, Stemmann C, Satoskar AR, Sleckman BP, Glimcher LH: Distinct effects of T-bet in TH1 lineage commitment and IFN-gamma production in CD4 and CD8 T cells. Science 2002;295:338-342.

-37 Kiwamoto T, Ishii Y, Morishima Y, Yoh K, Maeda A, Ishizaki K, Iizuka T, Hegab AE, Matsuno Y, Homma S, Nomura A, Sakamoto T, Takahashi S, Sekizawa K: Transcription Factors T-bet and GATA-3 Regulate Development of Airway Remodeling. Am J Respir Crit Care Med 2006;174:142-151.

-38 Hwang ES, Szabo SJ, Schwartzberg L, Glimcher LH: T helper cell fate specified by kinase-mediated interaction of T-bet with GATA-3. Science 2005;21:430-433.

39 Finotto S, De Sanctis GT, Lehr, Herz U, Buerke M, Schipp M, Bartsch B, Atreva R, Schmitt E, Galle PR, Renz H, Neurath MF: Treatment of allergic airway inflammation and hyperresponsiveness by antisense-induced local blockade of GATA-3 expression. J Exp Med 2001;193:1247-1260. 